Editorial

\title{
Tumor lysis syndrome caused by therapeutic monoclonal antibodies approved by FDA
}

Volume 5 Issue 4 - 2017

\section{Editorial}

In 1975, Monoclonal antibody (mAb) technique was created by Georges Köhler, César Milstein, and Niels Kaj Jerne by using mouse $\mathrm{x}$ mouse hybridoma, they shared the Nobel Prize in Physiology or Medicine in 1984 for the discovery. 8 years later, in 1992 FDA approved first therapeutic mAb Muromonab-CD3 (trade name Orthoclone OKT3) to reduce acute rejection in patients with organ transplants, since then, as of May 31, 2017, FDA has approved 70 therapeutic mAbs. ${ }^{1-3}$ Among them 28 were approved for treatment of cancers (Table 1). ${ }^{4-31}$ These therapeutic mAb targets at components expressed on cancer cell, possible mechanisms of cell lysis include complement-dependent cytotoxicity (CDC), antibody-dependent cell mediated cytotoxicity (ADCC), induced apoptosis, cancer cell growth inhibition, direct cytoxicities, and conjugates indirect effect resulting cancer cell death (radiation or internalized derives). Unfortunately, so far there is no tumor specific antigen (TSA) available for target, those antigens chosen as target, they also expressed at normal cells, which inevitably causes various adverse reactions, in this article, tumor lysis syndrome (TLS) is briefly reviewed.

TLS may be a consequence of liquid tumor treatment with therapeutic mAbs approved by FDA (Table 2), which is not seen in the labeling of therapeutic mAbs for solid tumor (Table 3). Acute renal failure, hyperkalemia, hypocalcemia, hyperuricemia, or hyperphosphatemia from tumor lysis, some fatal, can occur within 1224 hours after the first infusion of the therapeutic mAbs. Interestingly among those therapeutic $\mathrm{mABs}$ for liquid tumors, even targeting same antigen CD20, it may not cause TLS (Table 2). It is believed patients

\author{
Henry Hongrong Cai \\ Pharmacovigilance and drug safety, EMD Serono and InVentive \\ Health Clinical, USA
}

Correspondence: Henry Hongrong Cai,Ventive Health Clinical, 95 Cynthia Road Newton, MA 02459, USA, Tel 617-58I5161, Email hongrong.cai@yahoo.com

Received: January 01, 1970 | Published: June 06, 2017

with high tumor burden, high circulating lymphocyte count $(>25 \mathrm{x}$ $109 / \mathrm{L})$ or renal impairment are at greater risk for TLS and should receive appropriate tumor lysis prophylaxis with anti-hyperuricemics (e.g., allopurinol or rasburicase) and hydration prior to the infusion of the therapeutic mAb. Physicians should also consider leukoreduction with hydroxyurea or leukapheresis to reduce the peripheral white blood count to $<30,000 / \mu \mathrm{L}$ prior to administration of the therapeutic $\mathrm{mAb}$. During the initial days of treatment, monitor the laboratory parameters of patients considered at risk for TLS. For treatment of TLS, correct electrolyte abnormalities, monitor renal function and fluid balance, and administer supportive care, including dialysis as indicated.

Table I Monoclonal Antibodies for Cancer Therapy Approved By FDA.

\begin{tabular}{|c|c|c|c|c|c|}
\hline Drug name & Approval date & Company & Active integedients & Target & Indication \\
\hline Rituxan & II/26/I997 & IDEC & Rituximab & CD20 & B-NHL \\
\hline Herceptin & $9 / 25 / 1998$ & Genetech & Trastuzumab & EGF & Breast $\mathrm{Ca}$ \\
\hline Mylotarg & $5 / 17 / 2000$ & Wyeth & Gemtuzumab Ozogamicin & CD33 & AML \\
\hline Campath & $2 / 7 / 200 I$ & Genzyme & Alemtuzumab & CD52 & B-CLL \\
\hline Zevalin & $2 / 19 / 2002$ & Spectrum & Ibritumomab Tiuxetan & CD20 & B-NHL \\
\hline Erbitux & $2 / 12 / 2004$ & Imclone & Cetuximab & VEGFR & $\mathrm{MCC}$ \\
\hline Avastin & $2 / 26 / 2004$ & Genetech & Bevacizumab & VEGF & Colon $\mathrm{Ca}$ \\
\hline Vectibix & $9 / 27 / 2006$ & Amgen & Panitumumab & EGFR & Colorectal Ca \\
\hline Arzera & $10 / 26 / 2009$ & Glaxo & Ofatumumab & CD20 & $\mathrm{B}-\mathrm{CLL}$ \\
\hline Yervoy & $3 / 25 / 2011$ & BMS & Ipilimumab & CTLA-4 & Melanoma \\
\hline Adcetris & $8 / 19 / 2011$ & Seattle Sci & Brentuximab Vedotin & CD30 & $\mathrm{HL}$ \\
\hline Perjeta & $6 / 8 / 2012$ & Genetech & Pertuzumab & HER2 & Breast Ca \\
\hline Kadcyla & $2 / 22 / 2013$ & Genetech & Ado-Trastuzumab Emtansine & HER2 & Breast Ca \\
\hline Gazyva & $1 \mathrm{I} / \mathrm{I} / 2013$ & Genetech & Obinutuzumab & CD20 & B-CLL \\
\hline Cyramza & $4 / 21 / 2014$ & Eli Eilly & Ramucirumab & VEGFR2 & Gastric Ca \\
\hline Ketruda & $9 / 4 / 2014$ & MSD & Pembrolizumab & PD-I & Melanoma \\
\hline Bexxar & $12 / 3 / 20 \mid 4$ & Amgen & Tositumomab; lodine I I 3 I Tositumomab & CDI9+CD3 & ALL \\
\hline Opdivo & $|2 / 22 / 20| 4$ & BMS & Nivolumab & PD-I & Melanoma \\
\hline Unituxin & $3 / 10 / 2015$ & United Terap & Dinutuximab & GD2 & Neuroblastoma \\
\hline
\end{tabular}


Table Continued..

\begin{tabular}{llllll}
\hline Drug name & Approval date & Company & Active integedients & Target & Indication \\
\hline Darzalex & $11 / 16 / 2015$ & Janssen & Daratumumab & CD38 & MM \\
Portrazza & $11 / 24 / 2015$ & Eli Eilly & Necitumumab & EGFR & Lung cancer \\
Empliciti & $11 / 30 / 2015$ & BMS & Elotuzumab & SLAMF7 & MM \\
Tecentiq & $5 / 18 / 2016$ & Genetech & Atezolizumab & PD-LI & Urothelial Ca \\
Tecentriq & $10 / 18 / 2016$ & Genetech & Atezolizumab & PD-LI & Metastatic NSCLC \\
Lartruvo & $10 / 19 / 2016$ & Eli Eilly & Olaratumab & PDGFR- $\alpha$ soft tissue sarcoma \\
Bavencio & $3 / 23 / 2017$ & EMD serono & Avelumab & PD-LI & Metastatic Merkel Cell Ca \\
Imfinzi & $5 / 1 / 2017$ & Astrazeneca UK & Durvalumab & PD-LI & Metastatic Urothelial Ca \\
Bavencio & $5 / 9 / 2017$ & EMD serono & Avelumab & PD-LI & Metastatic Urothelial Ca \\
\hline
\end{tabular}

CD, cluster of differentiation; EGF, epidermal growth factor;VEGFR, vascular endothelial growth factor receptor;VEGF, vascular endothelial growth factor; EGFR, epidermal growth factor receptor; CTLA-4, cytotoxic t-lymphocyte-associated protein 4; HER2, human epidermal growth factor receptor 2; PD-I, programmed cell death protein I; GD2, glycolipid disialoganglioside; SLAMF7, signaling lymphocytic activation molecule family member 7; PD-LI, programmed death-ligand I; B-NHL, B-cell non-hodgkin's lymphoma; AML, acute myeloid leukemia; B-CLL, B-cell chronic lymphocytic leukemia; MCC, metastatic colorectal carcinoma; HL, hodgkin's lymphoma; ALL, acute lymphocytic leukemia; MM, multiple myeloma; PDGFR- $\alpha$, platelet-derived growth factor receptor alpha; MCC, merkel cell carcinoma

Table 2 Therapeutic Monoclonal Antibodies for Liquid Tumor Therapy Approved By FDA

\begin{tabular}{|c|c|c|c|c|c|c|}
\hline Drug name & Approval date & Company & Active integedients & Target & Indication & TLS \\
\hline Rituxan & I I/26/I997 & IDEC & Rituximab* & CD20 & $\mathrm{B}-\mathrm{NHL}$ & Yes \\
\hline *Mylotarg & $5 / 17 / 2000$ & Wyeth & Gemtuzumab Ozogamicin & CD33 & AML & Yes \\
\hline Campath & $2 / 7 / 2001$ & Genzyme & Alemtuzumab & CD52 & B-CLL & No \\
\hline Zevalin** & $2 / 19 / 2002$ & Spectrum & Ibritumomab Tiuxetan & CD20 & B-NHL & No \\
\hline Arzerra* & $10 / 26 / 2009$ & Glaxo & Ofatumumab & CD20 & B-CLL & Yes \\
\hline Adcetris* & $8 / 19 / 2011$ & Seattle Sci & Brentuximab Vedotin & CD30 & $\mathrm{HL}$ & Yes \\
\hline Gazyva* & $1 \mathrm{I} / \mathrm{I} / 20 \mathrm{I} 3$ & Genetech & Obinutuzumab & CD20 & B-CLL & Yes \\
\hline Darzalex & $1 \mathrm{I} / 16 / 2015$ & Janssen & Daratumumab & CD38 & MM & No \\
\hline Empliciti & $1 \mathrm{I} / 30 / 2015$ & BMS & Elotuzumab & SLAMF7 & MM & No \\
\hline Bexxar & $|2 / 3 / 20| 4$ & Amgen & Tositumomab; lodine I | 3 | Tositumomab & CDI9+CD3 & ALL & $\mathrm{N} / \mathrm{A}$ \\
\hline
\end{tabular}

Table 3 Therapeutic Monoclonal Antibodies for Solid Tumor Therapy Approved By FDA

\begin{tabular}{|c|c|c|c|c|c|c|}
\hline Drug name & Approval date & Company & Active integedients & Target & Indication & TLS \\
\hline Herceptin & $9 / 25 / 1998$ & Genetech & Trastuzumab** & EGF & Breast $\mathrm{Ca}$ & No \\
\hline Erbitux & $2 / 12 / 2004$ & Imclone & Cetuximab & VEGFR & MCC & No \\
\hline Avastin $* *$ & $2 / 26 / 2004$ & Genetech & Bevacizumab & VEGF & Colon $\mathrm{Ca}$ & No \\
\hline Vectibix & $9 / 27 / 2006$ & Amgen & Panitumumab & EGFR & Colorectal Ca & No \\
\hline Yervoy** & $3 / 25 / 20 I I$ & BMS & Ipilimumab & CTLA-4 & Melanoma & No \\
\hline Perjeta** & $6 / 8 / 2012$ & Genetech & Pertuzumab & HER2 & Breast Ca & No \\
\hline Kadcyla** & $2 / 22 / 2013$ & Genetech & Ado-Trastuzumab Emtansine & HER2 & Breast Ca & No \\
\hline Cyramza** & $4 / 21 / 20 \mid 4$ & Eli Eilly & Ramucirumab & VEGFR2 & Gastric Ca & No \\
\hline Keytruda** & $9 / 4 / 2014$ & MSD & Pembrolizumab & PD-I & Melanoma & No \\
\hline Opdivo** & $12 / 22 / 2014$ & BMS & Nivolumab & PD-I & Melanoma & No \\
\hline Unituxin** & $3 / 10 / 2015$ & United Terap & Dinutuximab & GD2 & Neuroblastoma & No \\
\hline Portrazza** & II/24/2015 & Eli Eilly & Necitumumab & EGFR & Lung cancer & No \\
\hline
\end{tabular}

\section{Acknowledgments}

None.

\section{Conflicts of interest}

The authors declare no conflicts of interest.

\section{References}

1. Cai HH. Therapeutic Monoclonal Antibodies Approved by FDA in 2015 MOJ Immunology. 2016;3(2):87.

2. Cai HH. Molecular Pharmacovigilance: Safety Signal for Drug Modification. MOJ Immunology. 2014;1(5):25.

3. Cai HH. Risk Evaluation and Mitigation Strategy for Approved Therapeutic Antibodies. MOJ Immunology. 2014;1(5):28.
4. http://www.accessdata.fda.gov/drugsatfda_docs/label/2002/ rituide021902LBp1.pdf

5. http://www.accessdata.fda.gov/drugsatfda_docs/label/1998/ trasgen0925981b.pdf

6. http://www.accessdata.fda.gov/drugsatfda_docs/label/2000/21174lbl.pdf

7. http://www.accessdata.fda.gov/drugsatfda_docs/label/ biologics/103948-5036_campath_lbl.pdf

8. http://www.accessdata.fda.gov/drugsatfda_docs/label/2002/ ibriide021902LB.pdf

9. http://www.accessdata.fda.gov/drugsatfda_docs/label/2004/125084lbl. pdf

10. http://www.accessdata.fda.gov/drugsatfda_docs/label/2004/125085lbl. pdf 
11. http://www.accessdata.fda.gov/drugsatfda_docs/ label/2006/125147s0000lbl.pdf

12. http://www.accessdata.fda.gov/drugsatfda_docs/label/2009/125326lbl. pdf

13. http://www.accessdata.fda.gov/drugsatfda_docs/ label/2011/125377s00001bl.pdf

14. http://www.accessdata.fda.gov/drugsatfda docs/ label/2011/125388s000,125399s000lbl.pdf

15. http://www.accessdata.fda.gov/drugsatfda_docs/label/2012/125409lbl. pdf

16. http://www.accessdata.fda.gov/drugsatfda_docs/label/2013/125427lbl pdf

17. http://www.accessdata.fda.gov/drugsatfda_docs $/$ label/2013/125486s000lbl.pdf

18. http://www.accessdata.fda.gov/drugsatfda_docs/label/2014/125477lbl. pdf

19. http://www.accessdata.fda.gov/drugsatfda_docs/label/2014/125514lbl. pdf

20. http://www.accessdata.fda.gov/drugsatfda docs/label/2003/ tosicor062703LB.pdf

21. http://www.accessdata.fda.gov/drugsatfda_docs/label/2014/125554lbl pdf
22. http://www.accessdata.fda.gov/drugsatfda_docs/ label/2015/125516s000lbl.pdf

23. http://www.accessdata.fda.gov/drugsatfda_docs/ label/2015/761036Orig1s000lbledt.pdf

24. http://www.accessdata.fda.gov/drugsatfda docs/ label/2015/125547s000lbl.pdf

25. http://www.accessdata.fda.gov/drugsatfda docs/ label/2015/761035s0001bl.pdf

26. http://www.accessdata.fda.gov/drugsatfda docs/ label/2016/761034Orig1s000lbl.pdf

27. https://www.accessdata.fda.gov/drugsatfda_docs/ label/2016/761041s000lbl.pdf

28. https://www.accessdata.fda.gov/drugsatfda_docs/label/2016/7610381bl. pdf

29. https://www.accessdata.fda.gov/drugsatfda_docs/ label/2017/761049s000lbl.pdf

30. https://www.accessdata.fda.gov/drugsatfda docs/ label/2017/761069s000lbl.pdf

31. https://www.accessdata.fda.gov/drugsatfda docs/ label/2017/761078s000lbl.pdf 